\title{
FIXED POINT THEOREMS FOR COMPATIBLE MULTI-VALUED AND SINGLE-VALUED MAPPINGS
}

\author{
HIDEAKI KANEKO \\ Department of Mathematics and Statistics \\ old Dominion University \\ Norfolk, VA 23529 U.S.A. \\ AND \\ SALVATORE SESSA \\ Universita di Napoli; Facolta di Architettura \\ Instituto Mathematics; Via Monteoliveto 3, \\ 80134 Napoli, Italy \\ (Received July 1, 1987 and in revised form October 1, 1987)
}

\begin{abstract}
The notion of compatibility for point-to-point mappings recently defined by Jungck is generalized to include multi-valued mappings. This idea is used to establish a fixed point theorem for a generalized contractive multi-valued mapping and a single-valued mapping.
\end{abstract}

KEY WORDS AND PHRASES: Compatible Multi-Valued Mappings, Fixed Point. 1980 AMS Subject Classification: 47H10 54H25

1. INTRODUCTION Jungck [2] proved the following theorem for f-contractive point-to-point mappings.

THEOREM 1. A continuous self-mapping $f$ of a complete metric space $(X, d)$ has a fixed point iff there exists a mapping $g: X \rightarrow X$ which commutes with $\mathrm{f}$ and such that $g(X) \subseteq f(X)$, $d(g x, g y) \leq h d(f x, f y)$ for all $x, y \in X$, where $0 \leq h \leq 1$. Futhermore, $f$ and $g$ have a unique common fixed point.

The present authors generalized this theorem in two different directions. Sessa [8], generalizing the notion of commutativity for point-to- point mappings, established the idea of weak commutativity for two self- mappings $\mathrm{f}$ and $\mathrm{g}$ of a metric space $(\mathrm{X}, \mathrm{d})$, i.e. $d(f g x, g f x) \leq$ $d(f x, g x)$ for all $\mathrm{x}$ in $\mathrm{X}$. Under this concept, he extended theorem 1. On the other hand, Kaneko [5] proved that theorem 1 can be extended to the setting of multi-valued mappings, generalizing a famous result of Nadler [7]. Recently Jungck [3] made an extension of weak commustivity in the following way. 
DEFINITION 1. Two self-mappings $f$ and $g$ of metric space $(X, d)$ are compatible iff $d\left(f g x_{n}, g f x_{n}\right) \rightarrow 0$ whenever $x_{n}$ is a sequence in $\mathrm{X}$ such that $f x_{n} \rightarrow t, g x_{n} \rightarrow t$ for some point $\mathrm{t}$ in $\mathrm{X}$.

It can be seen that two weakly commuting mappings are compatible but the converse is false. Examples supporting this fact can be found in [3].

The purpose of this paper is to extend the definition of compatibility to include multivalued mappings.

2. RESULTS Let $(X, d)$ be a metric space and $C B(X)$ the family of all closed bounded subsets of $X$. Let $H$ be the Hausdorff metric on $C B(X)$ induced by d, i.e.

$$
H(A, B)=\max \{\sup d(x, B): x \epsilon A, \sup d(x, A): x \epsilon B\}
$$

for all $\mathrm{A}, \mathrm{B}$ in $\mathrm{CB}(\mathrm{X})$, where $d(x, A)=\inf \{d(x, y): y \epsilon A\}$. It is well known $[10]$ that $(\mathrm{CB}(\mathrm{X}), \mathrm{H})$ is a metric space. It is indeed a complete metric space in the event that $(\mathrm{X}, \mathrm{d})$ is also a complete metric space.

DEFINITION 2. The mappings $f: X \rightarrow X$ and $T: X \rightarrow C B(X)$ are compatible iff $f T x \epsilon C B(X)$ for all $x \epsilon X$ and $H\left(T f x_{n}, f T x_{n}\right) \rightarrow 0$ whenever $x_{n}$ is a sequence in $\mathrm{X}$ such that $T x_{n} \rightarrow M \epsilon C B(X)$ and $f x_{n} \rightarrow t \epsilon M$.

Definition 2 extends definition 1 above. We are now in a position to prove our results.

Theorem 2. Let (X,d) be a complete metric space, $f: X \rightarrow X$ and $T: X \rightarrow C B(X)$ be compatible continuous mappings such that $T(X) \subseteq f(X)$ and

$$
H(T x, T y) \leq h \max \left\{d(f x, f y), d(f x, T x), d(f y, T y), \frac{1}{2}[d(f x, T y)+d(f y, T x)]\right\}
$$

for all $\mathrm{x}, \mathrm{y}$ in $\mathrm{X}$, where $0 \leq h<1$. Then there exists a point $t \epsilon X$ such that ft $\epsilon$ Tt.

Proof We draw inspiration from [6]. Let $x_{0} \in X$ be arbitrary and choose $x_{1} \in X$ such that $f x_{1} \epsilon T x_{0}$. This is possible since $T x_{0} \subseteq f(X)$. If $h=0$, then $d\left(f x_{1} T x_{1}\right) \leq H\left(T x_{0}, T x_{1}\right)=0$, i.e. $f x_{1} \epsilon T x_{1}$ since $T x_{1}$ is closed. Now assume that $h>0$ and for $k=\frac{1}{\sqrt{h}}>1$, by the definition of $\mathrm{H}$, there exists a point $y_{1} \epsilon T x_{1}$ such that $d\left(y_{1}, f x_{1}\right) \leq k H\left(T x_{1}, T x_{0}\right)$ (this is not generally true if $k \leq 1$ as it is seen in [7, remark at p. 480]). Since $T x_{1} \subseteq f(X)$, let $x_{2} \in X$ be such that $y_{1}=f x_{2}$. In general, if $x_{n}$ has been selected, choose $x_{n+1} \epsilon X$ so that $y_{n}=f x_{n+1} \epsilon T x_{n}$ and $d\left(y_{n}, f x_{n}\right) \leq k H\left(T x_{n}, T x_{n-1}\right)$ for each $n \geq 1$. Using (1), we have that

$$
\begin{aligned}
d\left(f x_{n}, f x_{n+1}\right) & \leq k H\left(T x_{n-1}, T x_{n}\right) \\
& \leq \sqrt{h} \max \left\{d\left(f x_{n-1}, f x_{n}\right), \frac{1}{2}\left[d\left(f x_{n-1}, f x_{n}\right)+d\left(f x_{n}, f x_{n+1}\right)\right]\right\} \\
& \leq \sqrt{h} \max \left\{d\left(f x_{n-1}, f x_{n}\right), d\left(f x_{n}, f x_{n+1}\right)\right\}
\end{aligned}
$$


i.e. $d\left(f x_{n}, f x_{n+1}\right) \leq \sqrt{h} d\left(f x_{n}, f x_{n-1}\right)$ for each n. Since $\sqrt{h}<1$, this shows that $\left\{f x_{n}\right\}$ is a Cauchy sequence, hence it coverges to some point $t \epsilon X$ using the completeness of $\mathrm{X}$. Furthermore, the above inequalities also show that $H\left(T x_{n-1}, T x_{n}\right) \leq h d\left(f x_{n-1}, f x_{n}\right)$. Since $\left\{f x_{n}\right\}$ is Cauchy, this must imply that $\left\{T x_{n}\right\}$ is a Cauchy sequence in the complete metric space $(\mathrm{CB}(\mathrm{X}), \mathrm{H})$. Now let $T x_{n} \rightarrow M \epsilon C B(X)$. Thus

$$
\begin{aligned}
d(t, M) & \leq d\left(t, f x_{n}\right)+d\left(f x_{n}, M\right) \\
& \leq d\left(t, f x_{n}\right)+H\left(T x_{n-1}, M\right) \rightarrow 0 \text { as } n \rightarrow \infty
\end{aligned}
$$

Since $\mathrm{M}$ is closed, $t \in M$ and the compatibility of $\mathrm{f}$ and $\mathrm{T}$ implies that $H\left(T f x_{n}, f T x_{n}\right) \rightarrow 0$ as $n \rightarrow \infty$. This along with the continuities of $\mathrm{f}$ and $\mathrm{T}$ imply that

$$
\begin{aligned}
d(f t, T t) & \leq d\left(f t, f f x_{n+1}\right)+d\left(f f x_{n+1}, T t\right) \\
& \leq d\left(f t, f f x_{n+1}\right)+H\left(f T x_{n}, T t\right) \\
& \leq d\left(f t, f f x_{n+1}\right)+H\left(f T x_{n}, T f x_{n}\right)+H\left(T f x_{n}, T t\right) \rightarrow 0
\end{aligned}
$$

as $n \rightarrow \infty$, i.e. fteTt since $\mathrm{Tt}$ is closed.

Q.E.D.

Remark 1. A nonempty subset $\mathrm{S}$ of $\mathrm{X}$ is proximinal if, for each $x \in X$, there exists a point $y \epsilon S$ such that $d(x, y)=d(x, S)$. Let $\mathrm{PB}(\mathrm{X})$ be the family of all bounded proximinal subsets of $\mathrm{X}$. If $T: X \rightarrow P B(X)$, then the interative process $\left\{y_{n}\right\}$ in the above proof can be simplified in the following way: if $x_{n}$ has been selected, let $x_{n+1} \epsilon X$ be such that $y_{n}=f x_{n+1} \epsilon T x_{n}$ and $d\left(f x_{n}, y_{n}\right)=d\left(f x_{n} T x_{n}\right)$. Note that an interation scheme of Smithson [9], where $T x$ is compact (hence proximinal) for all $x \epsilon X$, is included here. Since a proximinal set is closed, we have $P B(X) \subseteq C B(X)$. The results of $[4]$ and $[5]$ follow as corollaries.

COROLLARY 1. Let $(\mathrm{X}, \mathrm{d})$ be a complete metric space, $f: X \rightarrow X$ and $T: X \rightarrow$ $P B(X)$ be continuous mappings such that $f T x \epsilon P B(X)$ and $H(T f x, f T x) \leq d(f x, T x)$ for all $x \in X$. If (1) is satisfied for all $\mathrm{x}, \mathrm{y}$ in $\mathrm{X}, 0 \leq h<1$, and $T(X) \subseteq f(X)$, then there exists $t \in X$ such that $f t \epsilon T$.

COROLLARY 2. Let $(\mathrm{X}, \mathrm{d})$ be a complete metric space, $T: X \rightarrow C B(X)$ and $\mathrm{f}$ be a continuous self-mapping of $\mathrm{X}$ such that $H(T x, T y) \leq h d(f x, f y)$ and for all $\mathrm{x}, \mathrm{y}$ in $\mathrm{X}$, where $0 \leq h<1$ and $T f x=f T x$. If $T(X) \subseteq f(X)$, then there exists $t \epsilon X$ such that $f t \epsilon T t$.

Note that the continuity of $\mathrm{f}$ implies the continuity of $\mathrm{T}$ in corollary 2 . The following example shows theorem 2 is indeed a proper generalization over corollaries 1 and 2 .

EXAMPLE Let $X=[1, \infty)$ be endowed with the Euclidean metric d. Let $f x=2 x^{4}-1$ and $T x=\left[1, x^{2}\right]$ for each $x \geq 1$. T and $f$ are clearly continuous and $T(X)=f(X)=X$. Since $f x_{n} \rightarrow 1$ and $T x_{n} \rightarrow\{1\}$ iff $x_{n} \rightarrow 1, H\left(f T x_{n} T f x_{n}\right)=2\left(x_{n}^{4}-1\right) \rightarrow 0$ iff $x_{n} \rightarrow 1, f T x=$ 
$\left[1,\left(2 x^{4}-1\right)^{2}\right]$ for all $x \geq 1, \mathrm{f}$ and $\mathrm{T}$ are compatible. Since $H(T x, T y)=\left|x^{2}-y^{2}\right| \leq 2\left|x^{2}-y^{2}\right| \mid$ $x^{2}+y^{2} \mid / 2=d(f x, f y) / 2$, all the conditions of theorem 2 hold with $h=\frac{1}{2}$, but the above corollaries are not applicable since $\mathrm{f}$ and $\mathrm{T}$ do not weakly commute (for $x=2$ ), hence do not commute either.

In the sequel, we use the following lemma which is a slight generalization of proposition 2.2 (part 1) of $[3]$.

LEMMA. Let $T: X \rightarrow C B(X)$ and $f: X \rightarrow X$ be compatible. If $f w \epsilon T w$ for some $w \epsilon X$, then $f T w=T f w$.

PROOF. Let $x_{n}=w$ for each $n$. Then $f x_{n}=f w \rightarrow f w$ and $T x_{n} \rightarrow M \equiv T w$. Hence if $f w \epsilon T w$, then $H(f T w, T f w)=H\left(f T x_{n}, T f x_{n}\right) \rightarrow 0$ by the compatibility. Hence we must have $f T w=T f w$.

Q.E.D.

In order to obtain a fixed point result, we need additional assumptions as those given in [4] and [5].

THEOREM 3. Let $\mathrm{f}$ and $\mathrm{T}$ have the same meanings as in theorem 2. Assume also that for each $x \epsilon X$ either (i) $f x \neq f^{2} x$ implies $f x \notin T x$ or (ii) $f x \epsilon T x$ implies that $f^{n} x \rightarrow z$ for some $z \in X$. Then $\mathrm{f}$ and $\mathrm{T}$ have a common fixed point in $\mathrm{X}$.

PROOF. By Theorem 2, fteTt for some $t \epsilon X$ and $f T t=T f t$ by Lemma. Assuming (i), we deduce that $f t=f^{2} t \epsilon f T t=T f t$. Assuming (ii), it is clear that $f z=z$ by the continuity of f. We claim that $f^{n} t \epsilon T f^{n-1} t$ for each $\mathrm{n}$. To see this, we have that $f^{2} t=f f t \epsilon f t=T f t$. By lemma $(w=f t), f^{3} t=f f^{2} t \epsilon f T f t=T f^{2} t$. Repeating this argument, we obtain $f^{n} t \epsilon T f^{n-1} t$ and the continuity of $\mathrm{T}$ implies that

$$
d(z, T z) \leq d\left(z, f^{n} t\right)+d\left(f^{n} t, T z\right) \leq d\left(z, f^{n} t\right)+H\left(T f^{n-1}, T z\right) \rightarrow 0,
$$

i.e. $z \epsilon T z$ since $\mathrm{Tz}$ is closed. Hence $\mathrm{z}$ is a common fixed point of $\mathrm{f}$ and $\mathrm{T}$.

Q.E.D.

REMARK 2. Simple examples prove that the conditions " $T(X) \subseteq f(X)$ " and the compatibility of $f$ and $T$ are necessary in theorem 2. Unfortunately, it is not yet known if the continuity of both mappings $f$ and $T$ is necessary in theorem 2. However in the case that $f$ and $T$ are single-valued mappings, it suffices only the continuity of at least one of them. Moreover, the inequality (1) can be weakened as it is proved in the following results, which extends theorem 2.1 of $[1]$.

THEOREM 4. Let $(\mathrm{X}, \mathrm{d})$ be a complete metric space and $f, T: X \rightarrow X$ be two compatible mappings (def.1) such that $T(X) \subseteq f(X)$ and

$$
d(T x, T y) \leq H \max \{d(f x, f y), d(f x, T x), d(f y, T y), d(f x, T y), d(f y, T x)\}
$$

for all $\mathrm{x}, \mathrm{y}$ in $\mathrm{X}$, where $0 \leq h<1$. If one of $\mathrm{f}$ or $\mathrm{T}$ is continuous, then there exists a unique common fixed point of $f$ and $T$. 
PROOF. As in [1], it is seen that the sequence $\left\{T x_{n}\right\}$ where $T x_{n}=f x_{n+1}$ for each $\mathrm{n}$, is a Cauchy sequence. Hence it converges to some point $z \epsilon X$. If $\mathrm{T}$ is continuous, then $T^{2} x_{n} \rightarrow T z$ and $T f x_{n} \rightarrow T z$. Since $d\left(f T x_{n}, T f x_{n}\right) \rightarrow 0$ by compatibility, $f T x_{n} \rightarrow T z$, hence using (2),

$$
\begin{gathered}
d\left(T^{2} x_{n}, T x_{n}\right) \leq h \max \left\{d\left(f T x_{n}, f x_{n}\right), d\left(f T x_{n}, T^{2} x_{n}\right), d\left(f x_{n}, T x_{n}\right),\right. \\
\left.d\left(f T x_{n}, T x_{n}\right), d\left(f x_{n}, T^{2} x_{n}\right)\right\}
\end{gathered}
$$

which implies, as $n \rightarrow \infty$, that $d(z, T z) \leq h d(z, T z)$, i.e. $\mathbf{T} z=\mathbf{z}$. Since $T(X) \subseteq f(X)$, there exists a point $z$ ' such that $z=T z=f z^{\prime}$ and using (2) again,

$$
d\left(T^{2} x_{n}, T z^{\prime}\right) \leq h \max \left\{d\left(f T x_{n}, z\right), d\left(f T x_{n}, T^{2} x_{n}\right), d\left(z, T z^{\prime}\right), d\left(f T x_{n}, T z^{\prime}\right), d\left(z, T^{2} x_{n}\right)\right\}
$$

As $n \rightarrow \infty$, we deduce that $d\left(z, T z^{\prime}\right) \leq h d\left(z, T z^{\prime}\right)$, i.e. $z=T z^{\prime}=f z^{\prime}$ and by Lemma, $f z=f T z^{\prime}=T f z^{\prime}=T z=z$.

A similar proof can be made if we assume the continuity of $f$ instead of $T$. The uniqueness follows easily from (2).

Q.E.D.

REMARK 3. Note that our proof is different from that of $[1]$, in which it is proved that $f z=T z$ is the unique fixed point (only if $\mathrm{f}$ is continuous). In this paper, it is just the case that $f z=T z=z$.

REMARK 4. If neither $\mathrm{T}$ nor $\mathrm{f}$ is continuous, e.g. $X=[0,1], T 0=\frac{1}{4}, f 0=\frac{1}{2}, T x=$ $\frac{x}{8}, f x=\frac{x}{4}$ for $x \neq 0$, theorem 3 fails. Similar remarks can be made on the results of $[8]$.

ACKNOWLEDGEMENT: The authors would like to thank a referee for suggesting the formulation of definition 2 in its present form.

\section{REFERENCES}

1. K.M. Das and K.V. Naik, Common fixed point theorems for commuting maps on a metric space, Proc. Amer. Math. Soc. 77 (1979), 369-373.

2. G. Jungck, Commuting mappings and fixed points, Amer. Math. Monthly 83 (1976), 261-263.

3. G. Jungck, Compatible mappings and common fixed, points, Internat. J. Math. \& Math. Sci. 9 (1986), 771-779.

4. H. Kaneko, Single-valued and multi-valued f-contractions, Boll. Un. Mat. Ital. (6) 4-A (1985), 29-33.

5. H. Kaneko, A common fixed point of weakly commuting multi-valued mappings, submitted. 
6. T. Kubiak, Fixed point theorems for contractive type multi-valued mappings, Math. Japon. 30 (1985), 89-101.

7. S. Nadler, Multi-valued contraction mappings, Pacific J. Math. 20(2) (1969), 475-488.

8. S. Sessa, On a weak commutativity condition of mappings in fixed point considerations, Publ. Inst. Math. (Beograd) 32(46) (1982), 146-153.

9. R.E. Smithson, Fixed points for contractive multifunctions, Proc. Amer. Math. Sco. 27 (1971), 192-194. 


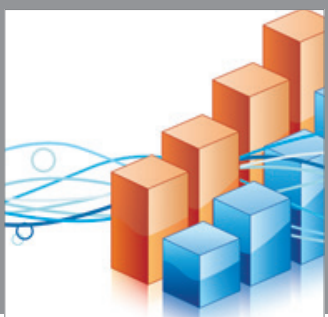

Advances in

Operations Research

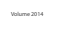

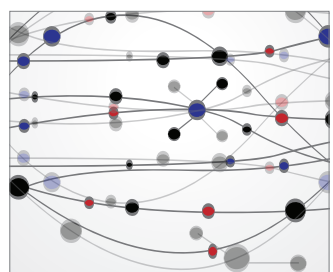

\section{The Scientific} World Journal
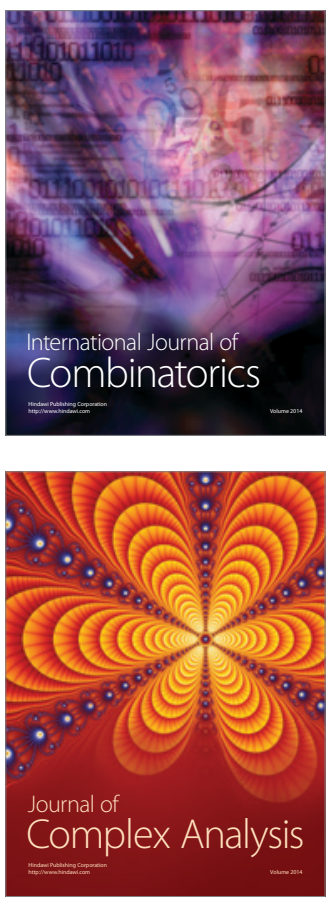

International Journal of

Mathematics and

Mathematical

Sciences
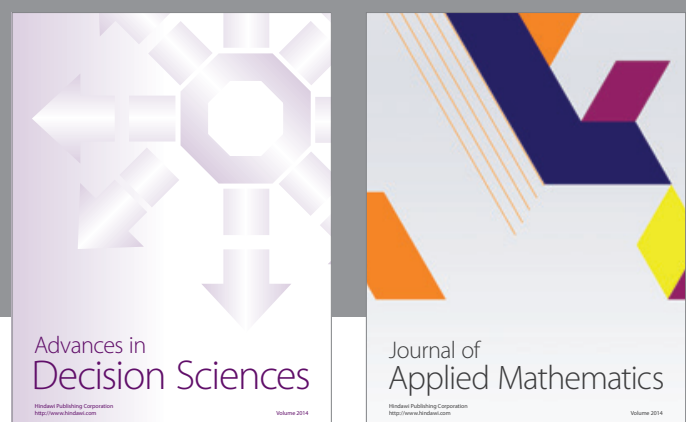

Journal of

Applied Mathematics
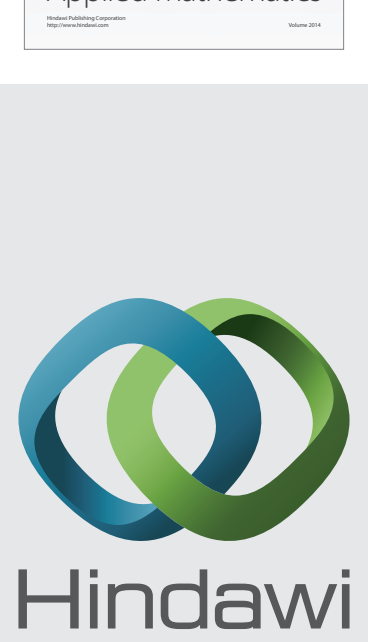

Submit your manuscripts at http://www.hindawi.com
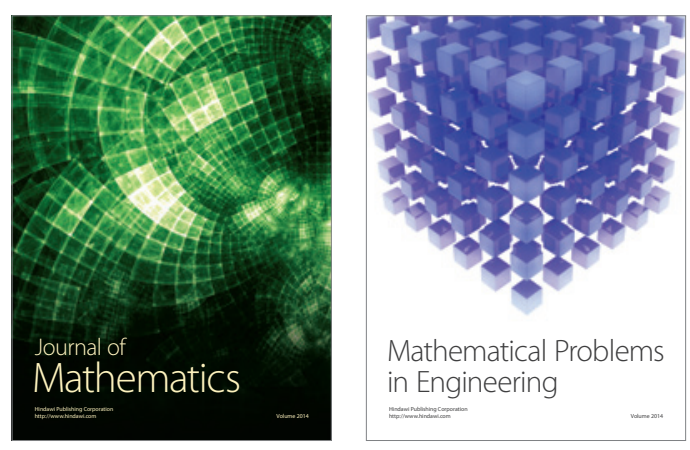

Mathematical Problems in Engineering
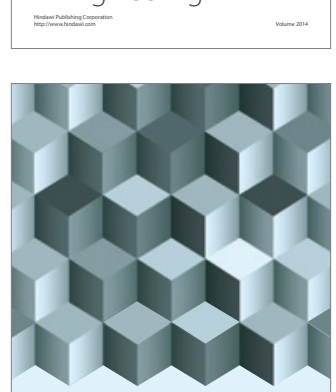

Journal of

Function Spaces
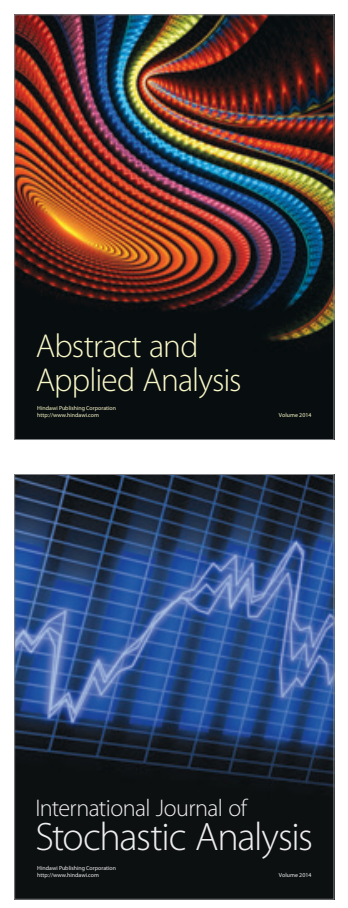

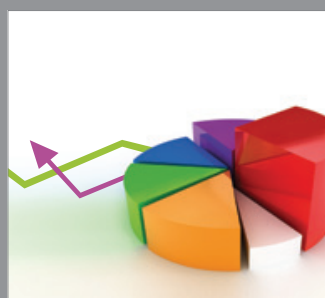

ournal of

Probability and Statistics

Promensencen
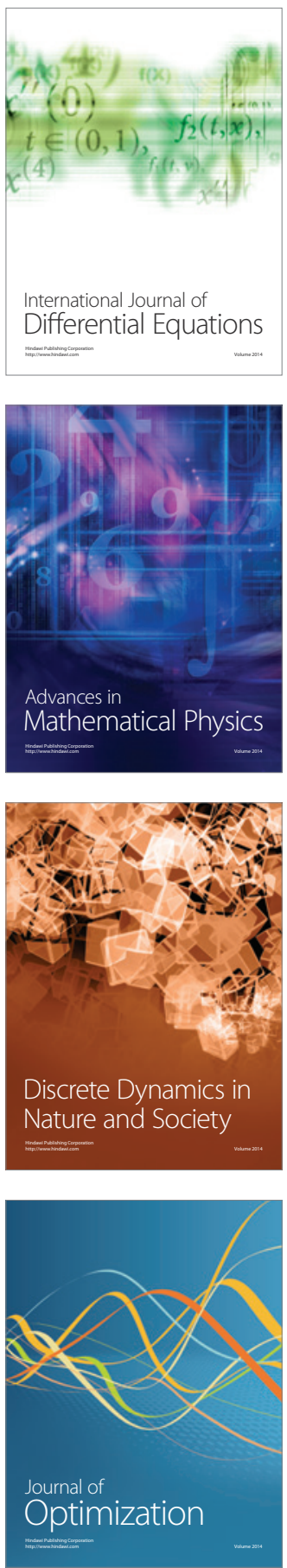